GENE PROFILING

\section{Validation on target}

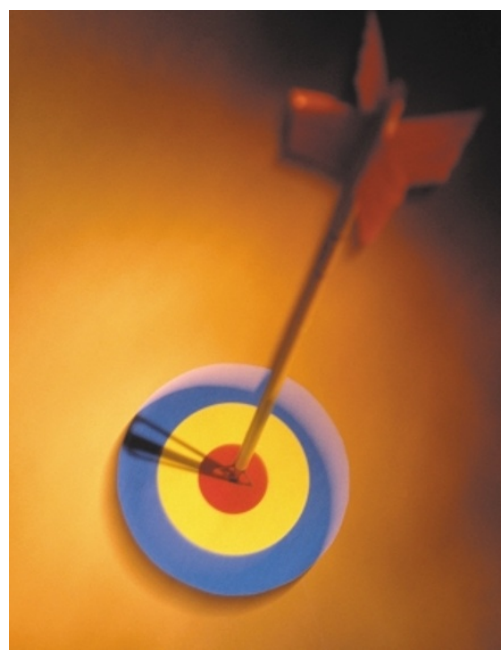

Multiple sclerosis (MS) is an autoimmune disease of the central nervous system that is characterized by progressive paralysis. Two types of histological lesion are associated with MS - acute lesions, which are characterized by inflammation, and chronic 'silent' ones that show scarring and demyelination. By doing microarray analysis on human biopsy samples, Lock and colleagues show, for the first time, that there are transcriptional differences between MS-associated lesions. Using a mouse model of MS, the authors also validate some of these differentially expressed molecules as potential therapeutic targets.
In the first study, Lock et al. compared transcripts from the two lesion types and showed differential gene expression between them, discovering several genes not previously thought to be important in MS. Comparing tissues from individuals with and without MS revealed genes whose expression was increased in MS samples, among them were the immune response molecules, major histocompatibility complex class II and immunoglobulin G; complement molecules; B-cell and macrophagespecific molecules; adhesion molecules, integrin $\beta_{4}$ and P-selectin; and several pro-inflammatory cytokines. Expression of neuron-associated genes and those encoding proteins associated with myelin production was decreased, suggesting that the capacity for tissue repair is compromised in MS patients.
Some of these differentially regulated gene products were chosen by the authors to test in the experimental autoimmune encephalomyelitis (EAE) mouse model of MS, as potential therapeutic targets. The first study revealed that the immunoglobulin Fcy receptor was upregulated in chronic lesions. Satisfyingly, the authors saw that Fcyreceptor-knockout mice, in which EAE was induced, had less severe acute disease and no chronic disease, compared to wild-type mice with EAE. The first study also showed that granulocyte-colony-stimulating factor (G-CSF) was upregulated in acute lesions. The authors showed that early treatment of EAE mice with G-CSF decreased the severity of the disease but that later treatment had no effect, suggesting that G-CSF might be a regulatory molecule that naturally suppresses acute attacks.

\title{
Altering the course of disease
}

\begin{abstract}
Although amyotrophic lateral sclerosis (ALS) is one of the most common neuromuscular diseases worldwide, we know relatively little about its causes. Most ALS cases are sporadic, but research has focused on familial ALS (FALS) in a search for the genetic defects that underlie the disease. One such defect - mutations in the superoxide dismutase gene, SOD1 occur in $~ 20 \%$ of FALS cases. But FALS onset and severity can vary remarkably in families with the same SOD1 mutation. Now, Giess et al. report that additional mutations at $C N T F$ - which encodes a potent motor-neuron survival factor - is probably one of several modifiers of ALS. Their findings indicate that, without the neuro-protective activity of CNTF, mutant SOD1 can trigger ALS earlier in both sporadic and familial patients, as well as in mice with mutant copies of both genes.

ALS is a rapidly progressive, invariably fatal neurological disease that attacks the neurons that control voluntary movement. It most commonly strikes between 40 and 60 years of age, but not always, as exemplified by the family studied by Giess et al. Three members of this family carry an
\end{abstract}

exonic SOD1 mutation believed to disrupt SOD1 function. The mother developed ALS at 54 ; however, the son developed the disease at 25 and died 11 months later, while his sister remains unaffected at 35 . Giess et al. genotyped CNTF in this family because of its previous association with ALS, and found that the son was homozygous null for CNTF, his mother heterozygous and his sister wild type. They also genotyped eight sporadic cases, who had an early onset of ALS, and who also proved to be $\mathrm{CNTF}^{-/}$.

To check that this was not just a chance correlation, Giess et al. also studied motorneuron survival in mice with mutations in both genes. As previous studies of ALS had indicated that mutant SOD1 acts as a dominant gain of function, the authors crossed transgenic mice ( $h S O D-1 G 93 A)$ that overexpress a disease-associated, human mutant SOD1 with Cntf null mice. They found that $h S O D-1 G 93 A / C n t f^{-/-}$ mice lose more lumbar spinal-cord motor neurons and have an earlier onset of disease than $h S O D-1 G 93 \mathrm{~A} / \mathrm{Cntf} f^{+/+}$mice, but that disease duration is unchanged by Cntfloss. Linkage analysis showed that, although disease incidence is regulated by SOD1 in this cross, disease onset, when analysed as a quantitative trait, is controlled by Cntf.

One study has reported that $2 \%$ of healthy individuals are homozygous for a null CNTF mutation - indeed, the unaffected father of the proband reported here was $\mathrm{CNTF}^{+/}$. Understanding how such mutations combine with other spontaneous, and perhaps epigenetic, events during life to give rise to sporadic neurodegenerative

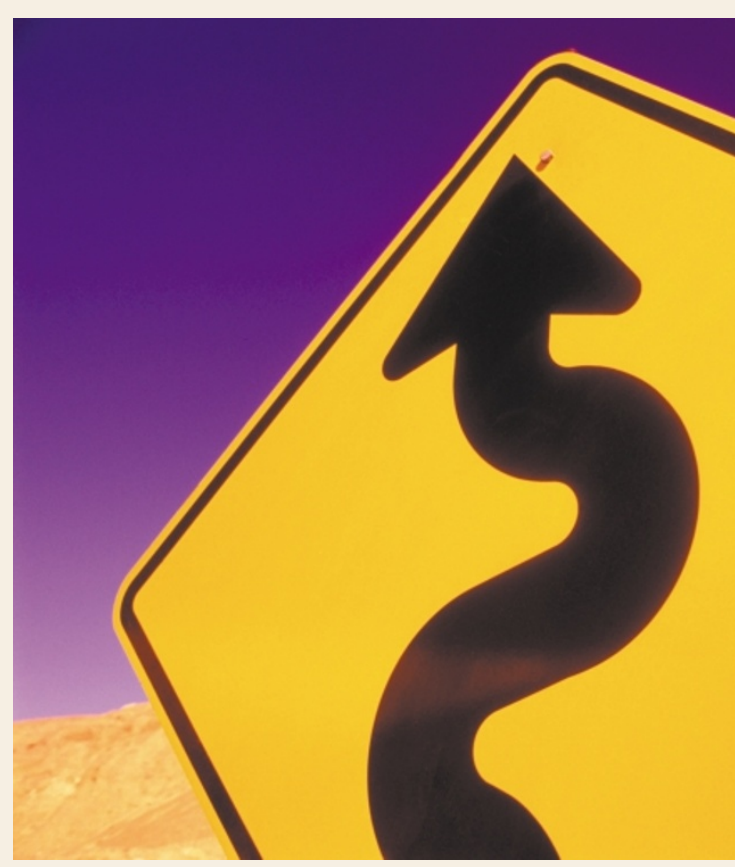


Although EAE is a useful model for MS, clinical trials have shown that many efficacious therapies in the mouse have not worked in humans. Therefore, caution must be exercised in spite of the validation in mice of the two reported MS targets. However, this study does provide proof of principle for using microarray technology to identify new therapeutic targets. Databanks that contain large microarray data sets generated by these types of study will allow more meaningful cross-data-set comparisons to get the most out of the increasing numbers of such analyses.

Melanie Brazil, Associate Editor, Nature Reviews Drug Discovery

(2) References and links ORIGINAL RESEARCH PAPER Lock, C. et al. Gene-microarray analysis of multiple sclerosis lesions yields new targets validated in autoimmune encephalitis. Nature Med. 8, 500-507 (2002)

disease should fuel research for years to come.

Jane Alfred

(2) References and links ORIGINAL RESEARCH PAPER Giess, R. et al. Early onset of severe familial amyotrophic lateral sclerosis with a SOD-1 mutation: potential impact of CNTF as a candidate modifier gene. Am. J. Hum. Genet. $\mathbf{7 0}$, 1277-1286 (2002)

WEB SITE

NINDS amyotrophic lateral sclerosis

information page: http://www.ninds.nih.gov/ health_and_medical/pubs/als.htm

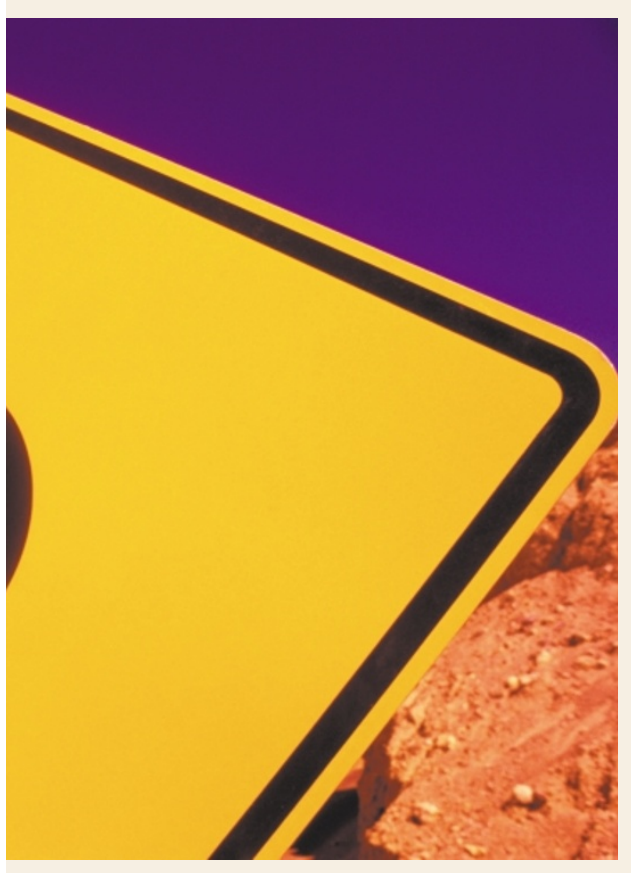

GENE EXPRESSION

\section{Peripheral oscillations}

Circadian rhythms are most often studied in association with behaviour and the underlying gene-expression changes in the brain. But circadian clocks that generate transcription periodicity also exist in many peripheral organs, as discussed in a new study published in Nature that demonstrates extensive circadian gene regulation in the hearts and livers of mice. In the biggest study of its kind, the authors show that this type of gene regulation is more extensive than previously thought and that, although it has a specialized role in a given tissue, it also has a broad biological function.

Although the fact that peripheral tissues have circadian regulation is not new, the extent of this regulation remained largely unknown. To investigate this, Storch et al. performed a microarray analysis on the hearts and livers of mice whose light/dark cycle had been synchronized. Using a set of genes with known circadian regulation as a guide, they found that $10 \%$ of liver genes and $8 \%$ of heart genes were regulated by the circadian clock. Although oscillations in gene expression were generally out of phase with each other in the liver, they were much more synchronized in the heart.

Interestingly, there was very little overlap between the genes that oscillate in the heart and in the liver - only 37 genes shared the same phase of peak expression. The authors point out that the core set of genes is important as it is likely to include those that are important for the functions of the peripheral clocks. Among them, they found genes involved in chromatin regulation, such as DNMT1-associated protein-1, and in ubiquitin pathways, suggesting that gene expression, in general, and protein stability are influenced by circadian rhythms.

To determine the kinds of processes that involve genes with circadian regulation, Storch and colleagues used a gene-ontology approach to sort the data into three categories: biological process, molecular function and cellular component. Their analysis indicated that, even in a single tissue, the circadian clock regulates many diverse processes, several of which are shared by the liver and the heart. So, the authors conclude that, although the genes regulated by circadian clocks are different in the two tissues, this type of regulation influences many related or overlapping processes.

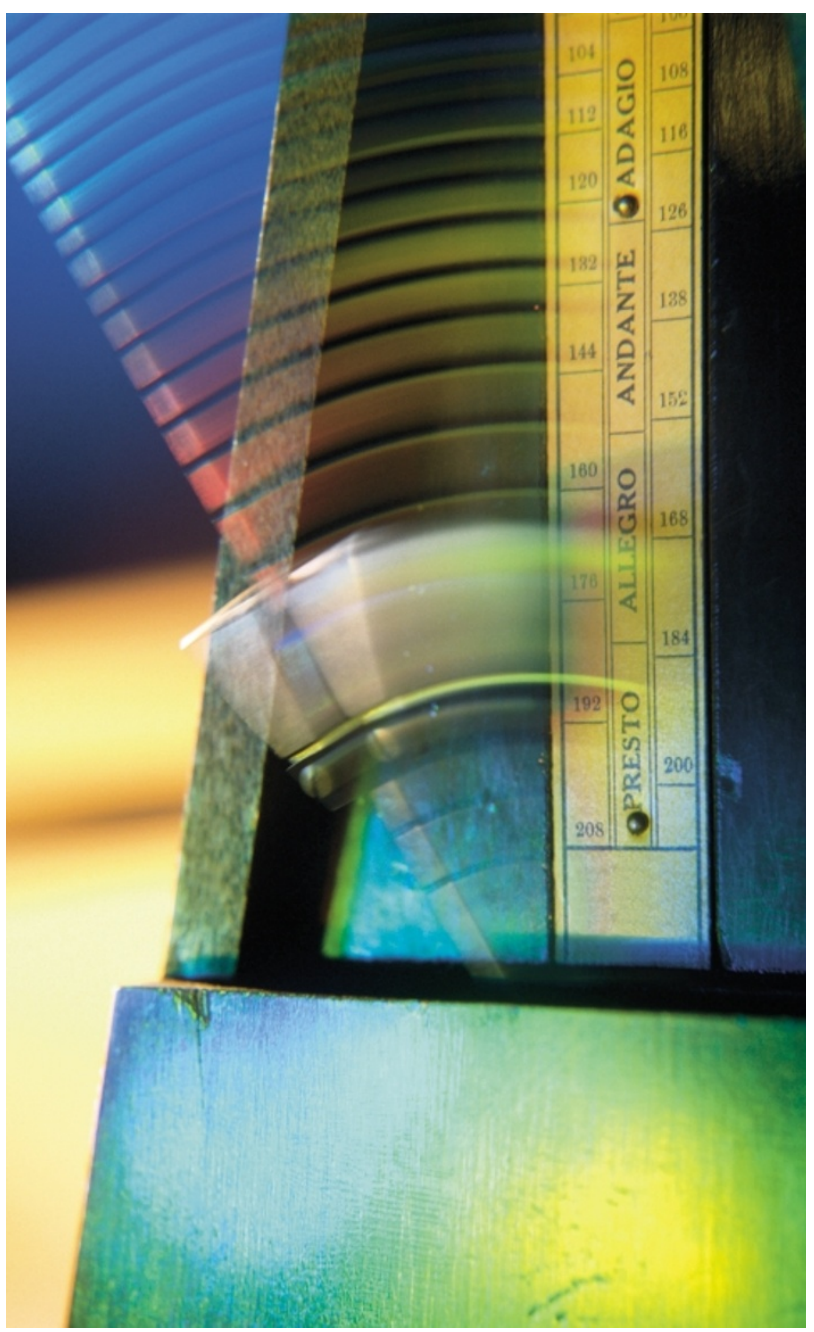

As the authors point out, there are some limitations to their study. They admit that transcript oscillations need not be reflected at the protein level, which depends, at least in part, on protein turnover. Also, for some proteins, circadian oscillations might have little physiological consequence. Nonetheless, together with another study published in Cell, which looked at circadian gene regulation in the liver and in a part of the hypothalamus that is known to harbour the 'pacemaker' of circadian control, this study substantially improves our understanding of circadian gene expression and provides evidence about the core clock mechanism that underlies it.

Magdalena Skipper

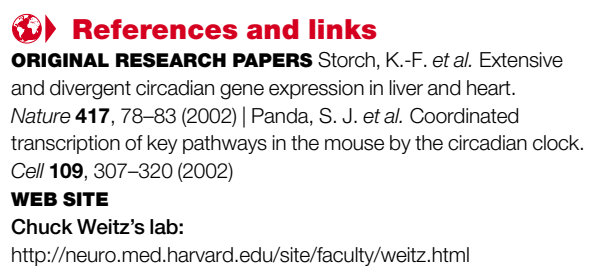

